\section{In vivo volumetric imaging of the human upper eyelid with ultrahigh-resolution optical coherence tomography}

\author{
Kostadinka Bizheva, ${ }^{\text {a }}$ Patrick Lee, ${ }^{\text {a }}$ Luigina Sorbara, ${ }^{\text {b }}$ \\ Natalie Hutchings, ${ }^{b}$ and Trefford Simpson ${ }^{b}$ \\ aniversity of Waterloo, Department of Physics and \\ Astronomy, 200 University Avenue West, Waterloo, \\ Ontario N2L3G1 Canada \\ ${ }^{\mathrm{b}}$ University of Waterloo, School of Optometry, 200 \\ University Avenue West, Waterloo, Ontario N2L3G1 Canada
}

\begin{abstract}
The upper eyelid is a biological tissue with complex structure, essential for the maintenance of an optically clear ocular surface due to its physical (blinking) effect. The Meibomian glands (MGs) are structures that lie beneath the surface of the inner eyelid and are partially responsible for the production of the superficial oily layer of the tear film. The MGs are only superficially visible under magnification when the eyelid is everted. We present for the first time in vivo 3-D images of healthy and inflamed human MGs. Tomograms were acquired from the tarsal plate of everted human eyelids with a 1060-nm ultrahigh-resolution optical coherence tomography (UHOCT) system, with $\sim 3 \mu \mathrm{m} \times 10 \mu \mathrm{m}$ (axial $\times$ lateral) resolution in biological tissue at the rate of 91,911 A-scans/s. Comparison with histology shows that the UHOCT images reveal a spatial distribution of structures that appear to correspond with the MGs' acini and ducts (in healthy subjects), and accumulation of heterogeneous, highly scattering biological material and clear fluids in the visibly blocked glands. Noninvasive, volumetric highresolution morphological imaging of the human tarsal area could have a significant impact in the clinical diagnosis of inflammatory and noninflammatory lid pathologies. ๑ 2010 Society of Photo-Optical Instrumentation Engineers. [DOI: 10.1117/1.3475957]
\end{abstract}

Keywords: optical coherence tomography; medical imaging; biomedical optics; human eyelid structure; Meibomian glands.

Paper 10340LR received Jun. 17, 2010; revised manuscript received Jul. 7, 2010; accepted for publication Jul. 8, 2010; published online Aug. 5, 2010.

The human upper eyelid has two principal roles: it acts as a protective barrier for the globe, closing the eye in response to prevent any potential penetration of a foreign body, and it also has a lubricating mechanism whereby the tear film covering the anterior surface of the eye is replenished by routine involuntary blinking. Located in the upper eyelid and sup-

Address all correspondence to: Kostadinka Bizheva, Department of Physics and Astronomy, University of Waterloo, 200 University Avenue West, Waterloo, ON N2L3G1, Canada. Tel: 519-888-4567x37517; Fax: 519-746-8115; E-mail: kbizheva@sciborg.uwaterloo.ca ported by the more rigid connective tissue of the tarsal plate are a number of glandular structures, consisting of $\sim 30-40$ Meibomian glands (MGs). The main ducts of the MGs are oriented perpendicular to and terminate at the line of the eyelashes. Each MG comprises clusters of acini branching from the main duct via fine ductules, ${ }^{1}$ resembling a grapevine. Epithelial cells in the acini synthesize and release lipids, which are secreted through the ducts onto the ocular surface. These lipids form the superficial layer of the tear film and are thought to slow down tear evaporation and lubricate the eyelids during blinking. ${ }^{2}$ Although the surface of the tarsal region of the human upper eyelid can be photographed using clinical devices such as a slit lamp, the volumetric distribution of the MG's ducts and acini has not yet been imaged in vivo. Detailed morphological images of individual human MG's acini acquired in vivo with laser confocal microscopy have been published recently, ${ }^{3}$ although these images do not reveal the volumetric structure of the MGs. Noninvasive, 3-D highresolution imaging of the human upper eyelid glandular structure could have significant impact on the clinical diagnostics of inflammatory and noninflammatory lid edema, hematomas, cysts, tumors, injuries, infections, congenital anomalies, eyelid adhesions, and disturbances in the position of the lid and in better understanding of the physiological response of the upper eyelid to these various conditions and causes. ${ }^{4}$

Optical coherence tomography (OCT) is an imaging modality that allows for volumetric, noninvasive imaging of the morphology of biological tissue with micrometer-scale resolution at imaging depths of 1 to $2 \mathrm{~mm}$ below the tissue surface. $^{5}$ Over the past 10 years, commercial and research grade ultrahigh-resolution OCT (UHROCT) systems have been used in ophthalmology to acquire noninvasively and in vivo high-resolution images of the human anterior chamber, specifically, the cornea. ${ }^{6,7}$ Here, we present, to our knowledge for the first time, in vivo 3-D UHROCT tomograms of the human tarsal plate in healthy subjects and an inflamed Meibomian gland of the human upper eyelid.

A high-speed, $1060 \mathrm{~nm}$ UHROCT system was developed for in vivo imaging of the human anterior and posterior eye. 8,9 When applied to imaging anterior structures of the human eye, this system has a major advantage over other UHROCT systems operating at $800 \mathrm{~nm}$ or $1300 \mathrm{~nm}$ : the acquired images do not require post-processing for dispersion compensation, since water has a dispersion null in the $1 \mu \mathrm{m}$ spectral region. Briefly, the UHROCT system is based on a compact fiber optic Michelson interferometer, connected to a superluminescent diode (SLD; Superlum Ltd.; $\lambda_{c}=1020 \mathrm{~nm}, \Delta \lambda$ $=110 \mathrm{~nm}$ ). The sample arm of the system is connected to an optical imaging probe, consisting of three achromat lenses and a pair of galvanometric scanners, mounted on a modified slit lamp biomicroscope. The interference signal is detected with a custom, high-performance spectrometer (P\&P Optica, Inc.), interfaced to a 1024 pixel linear array InGaAs camera (SUI, Goodrich Corp.) with $92 \mathrm{kHz}$ readout rate. The UHOCT system provides $3 \mu \mathrm{m}$ axial and $\sim 10 \mu \mathrm{m}$ lateral resolution in biological tissue and $99 \mathrm{~dB}$ SNR for $1.3 \mathrm{~mW}$ power of the imaging beam. Images are processed with MATLAB (Mathworks) and Amira (Visage Imaging, Inc.).

The 2-D and 3-D tomograms were acquired in vivo from

1083-3668/2010/15(4)/040508/3/\$25.00 ๑ 2010 SPIE 


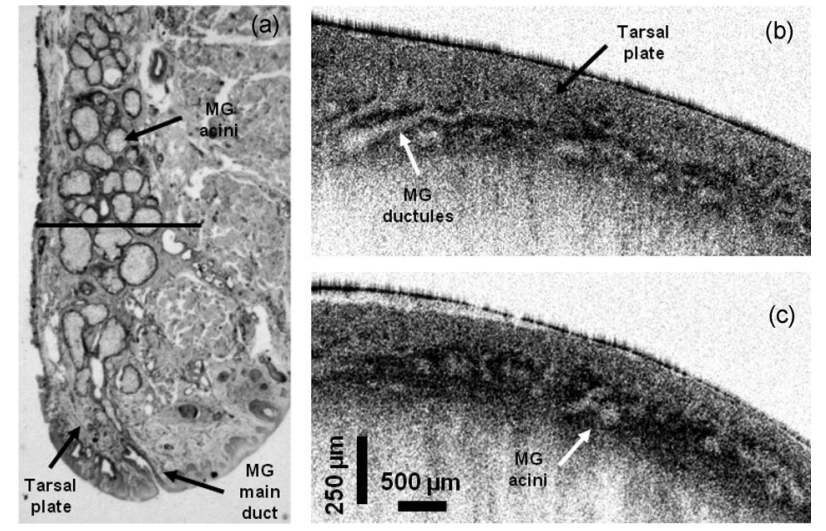

Fig. 1 Representative histological cross section (PAS staining) of the human upper eyelid (a) illustrating the morphology of the tarsal plate and a Meibomian gland (MG). The black line illustrates the orientation of the UHROCT B-scans. Representative UHROCT tomograms of the everted human upper eyelid with white arrows pointing to the lobular structures (c), connected to a ductule (b), likely corresponding to a MG acinus and ductule. The black arrow in Fig. 1(b) indicates the tarsal plate. Image dimensions $1000 \times 400$ (A-scans $\times$ pixels).

the everted eyelids of three human subjects. The imaging procedure was approved by the University of Waterloo Human Ethics Committee. Two of the subjects had normal, healthy eyelids, while the third had an inflamed MG (chalazion). The subjects were prescreened and their eyelids everted by a licensed optometrist. Volumetric images $(1000 \times 512 \times 256)$, corresponding to $4-\mathrm{mm} \times 1 \mathrm{~mm} \times 2 \mathrm{~mm}$ physical dimensions (horizontal $\times$ depth $\times$ vertical), were acquired from different locations in the eyelid, in direction from the fold toward the marginal area, perpendicular to the tissue surface and across the orientation of the MG's main ducts.

Figure 1 shows representative UHROCT 2-D images [(b) and (c)] of a healthy human eyelid. For comparative purposes, a representative upper eyelid histology (periodic acid schiff (PAS) staining) image is also shown (a). The UHROCT 2-D images were obtained perpendicular to the known anatomic course of the MG's main ducts, as illustrated by the black line on the histology image [Fig. 1(a)]. The UHROCT image dimensions were $1000 \times 400$ (A-scans $\times$ pixels), corresponding to 4 -mm lateral and $\sim 0.8-\mathrm{mm}$ depth scan. The tomograms show relatively homogeneous superficial tissue presumably, the inner surface connective tissue of the tarsal plate [Fig. 1(b), black arrow] and a network of low-reflective lobes with connecting ducts presumably cross sections of the MG acini [Fig. 1(c), white arrow] and ductules [Fig. 1(b), white arrow] adjacent to an additional area of relatively homogeneous tissue (presumably, outer surface connective tissue of the tarsal plate).

Two videos, presented in Fig. 2, show a cross-sectional view (a) and an en face view (b) of a representative volumetric image set of the human upper eyelid. The image sequence in Fig. 2(a) was acquired in the direction from the fold of the everted eyelid toward the eyelashes and exhibits cross sections of ductlike structures (low-reflective, dark brown, large circular features) and associated smaller irregularly shaped lobes (pale brown). The video in Fig. 2(b) shows the same structures in en face view. The lobelike structures appear to form clusters near the ends of the ductlike structures, leading

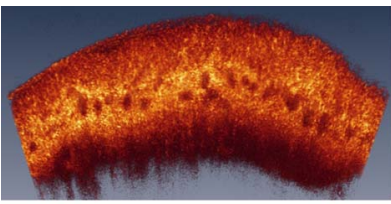

(a)

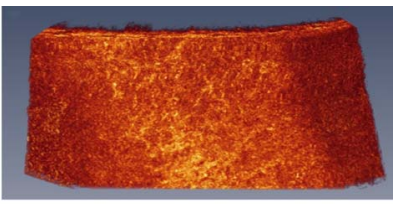

(b)
Fig. 2 Videos of the observed optical structure of a healthy human upper eyelid acquired in vivo. (a) Video 1 (QuickTime, 6.2 MB) the 3-D sequence was acquired across the MG's main duct, in the direction from the fold (first frame) toward the eyelashes (last frame) of the everted eyelid. [URL: http://dx.doi.org/10.1117/1.3475957.1]. (b) Video 2 (QuickTime, $8 \mathrm{MV}$ ) top view of the same image set showing the spatial distribution of the MG's ducts and the acini. Image dimensions $1000 \times 400 \times 256$. [URL: http://dx.doi.org/10.1117/ 1.3475957.2]. (Color online only.)

us to conclude that they likely correspond to the MG's acini and ductules, respectively, as seen in histological observations of the eyelid structure. The ductlike structures appear similar to large blood vessels, although there are major differences: the cross section of an MG's main duct increases in direction from the fold toward the eyelashes, and the main ducts of all MGs in the human eyelid are oriented parallel to each other, which corresponds to the morphological features observed in the en face tomograms [Fig. 2(b)].

Figure 3 shows a selection of representative en face images from the video in Fig. 2(b). The tomogram shown in Fig. 3(a) was acquired $\sim 200 \mu \mathrm{m}$ below the tissue surface, and there appears to be a large, ductlike structure [presumably the MG main duct; Fig. 3(b) black arrow]. To the temporo-lateral (right) side, there appears to be additional duct structure that is positioned deeper from the inner surface of the eyelid. On the naso-lateral (left) side of the large ductlike structure, a cluster of low-reflective (pale brown), irregularly shaped lobular features (presumably acini) appears, visible on the highly reflective background. A magnified $(2 \times)$ view of the section in Fig. 3(a) marked with the white rectangle is shown in Fig. 3(b), where the lobular features are better observed. The sequence of en face images [Figs. 3(c)-3(h)] obtained from different depths within the everted eyelid shows the spatial distribution of the ductlike and lobular features within the tissue.

Volumetric images were also acquired from a clinically diagnosed chalazion (nodular swelling in the tarsal plate), and representative tomograms are shown in Fig. 4. The images were acquired in the direction from the fold of the everted eyelid, across the visible, superficial mass of the chalazion, toward the eyelashes. Figure 4(a) shows the structural appearance of the relatively unaffected tissue adjacent to the mass of the chalazion. In Fig. 4(b), a small region of highly reflective tissue, enveloped in a thin membrane and surrounded by optically clear material (likely fluid; black arrow) is visible. Figure 4(c) shows a cystlike feature of almost round shape, with a thick membrane [Figs. 4(c) and 4(d); black arrow], filled with optically clear material, most likely fluid. The subsequent UHROCT tomograms acquired across the bulk of the swelling [Figs. 4(d)-4(f)] show that as the size of the mass increases, its shape becomes more irregular and it is filled with clusters of highly reflective material [Fig. 4(d); gray arrow]. On the basis of current knowledge of the histopathological mechanisms associated with this type of swelling, ${ }^{10}$ the 


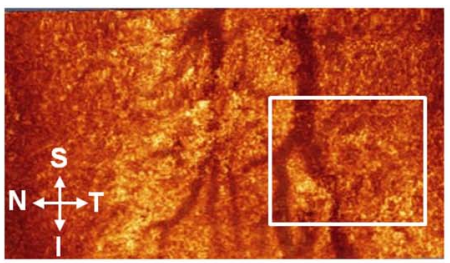

(a)

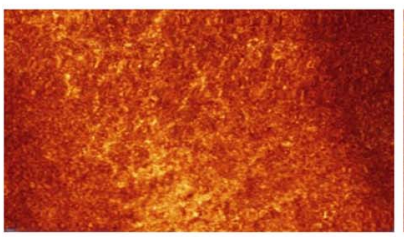

(c)

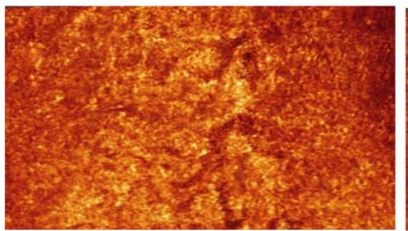

(e)

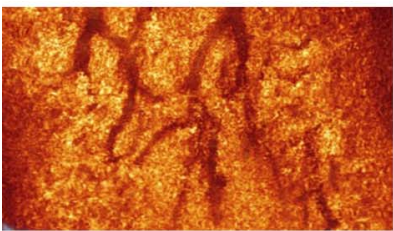

(g)

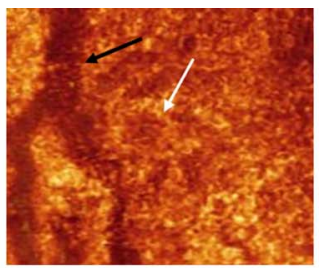

(b)

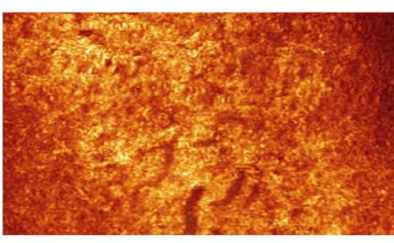

(d)

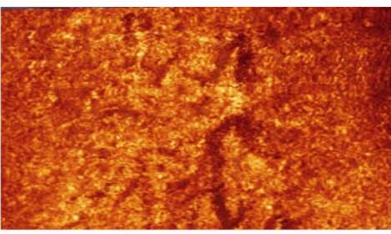

(f)

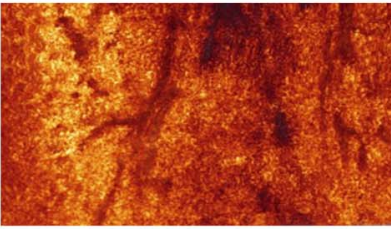

(h)
Fig. 3 Selected frames from the video in Fig. 2(b). (a) A UHROCT en face image, acquired $\sim 200 \mu \mathrm{m}$ below the inner surface of the human upper eyelid. (b) Enlarged $(2 \times)$ view of a ductlike (black arrow) and lobular structure, with the white arrow pointing at the location of an apparent acinus. (c) to (h) Sequential en face images of the upper eyelid's glandular structure, acquired at different depths below the tissue surface. Directions: $\mathrm{S}$-superior, I-inferior, $\mathrm{N}$-nasal, and T-temporal. (Color online only.)

observed highly reflective spots within the cyst may be agglomerations of inflammatory cells such as lymphocytes, epitheloid cells, or multinucleated giant cells, while the clear fluid may be liberated sebaceous material.

In summary, a state-of-the-art, high-speed, UHROCT system was used to acquire for the first time in vivo volumetric images of the everted human upper eyelid. The 3-D tomograms revealed the apparent intricate structure of the healthy Meibomian glands' ducts and acini, which were previously seen only with ex vivo histological analysis. This work also demonstrates the clinical relevance and potential of the UHOCT technology for noninvasive diagnostics of abnormalities in the upper eyelid, by revealing the internal structure of a chalazion, something previously requiring a biopsy.

\section{Acknowledgments}

The authors would like to thank J. Maram, S. Shakeel, and D. Lee for assistance with the data acquisition and image processing and T. F. Freddo for the eyelid histology slide. This work was supported in part by research grants from the Natu-

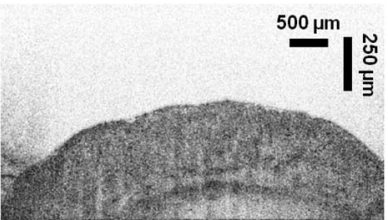

(a)

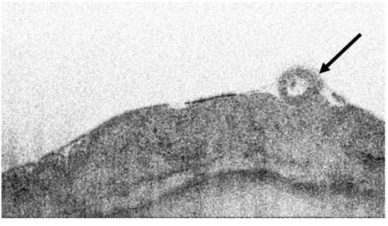

(c)

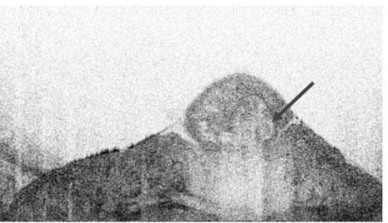

(e)

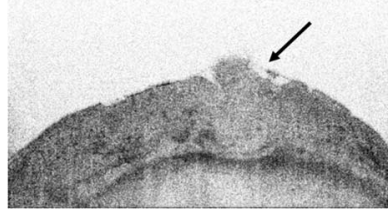

(b)

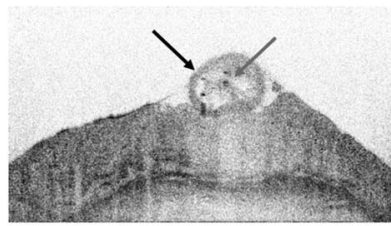

(d)

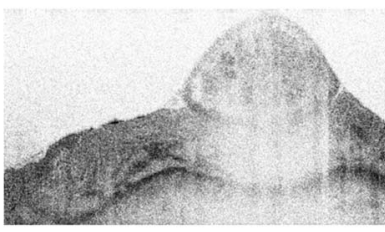

(f)
Fig. 4 Sequential UHROCT tomograms traversing a clinically diagnosed chalazion (inflamed MG), acquired in vivo from an everted left upper lid in the direction from the fold toward the eyelashes. Figure 4(a) shows the relatively normal tissue of the tarsal plate adjacent to the nodular swelling. (b) to (f) Sequential tomograms acquired across the mass of the nodular swelling exhibit a variety of cellular conformations enclosed in a membrane [(c) and (d), black arrows], filled with optically nonreflective/nonscattering material and also scattering content [(d) and (e), gray arrows].

ral Sciences and Engineering Research Council (NSERC) and the Canadian Institute for Health Research (CIHR).

\section{References}

1. J. J. Kanski, Clinical Ophthalmology, 4th ed., p. 12, ButterworthHeinemann, Oxford, UK (1999).

2. J. P. McCulley and W. E. Shine, "Meibomian gland function and the tear lipid layer," Ocul. Surf. 1, 97-106 (2003).

3. N. Efron, M. Al-Dossari, and N. Pritchard, "In vivo confocal microscopy of the palpebral conjunctiva and tarsal plate," Optom. Vision Sci. 86(11), E1303-E1308 (2009).

4. H. Pau, Differential Diagnosis of Eye Diseases, 2nd ed., C. F. Blodi, Ed., Thieme Medical Publishers, New York (1988).

5. D. Huang et al., "Optical coherence tomography," Science 254, 1178 (1991).

6. M. Gora, K. Karnowski, M. Szkulmowski, B. J. Kaluzny, R. Huber, A. Kowalczyk, and M. Wojikowski, "Ultra-high-speed swept-source OCT imaging of the anterior segment of human eye at $200 \mathrm{kHz}$ with adjustable imaging range," Opt. Express 17, 14880-14894 (2009).

7. J. Jungwirth, B. Baumann, M. Pircher, E. Götzinger, and C. K. Hitzenberger, "Extended in vivo anterior eye-segment imaging with full-range complex spectral domain optical coherence tomography," J. Biomed. Opt. 14(5), 050501 (2009).

8. P. Puvanathasan, P. Forbes, Z. Ren, D. Malchow, S. Boyd, and K. Bizheva, "High-speed, high-resolution Fourier-domain optical coherence tomography system for retinal imaging in the $1060 \mathrm{~nm}$ wavelength region," Opt. Lett. 33(21), 2479-2481 (2008).

9. N. Hutchings, T. L. Simpson, C. Hyun, A. A. Moayed, S. Hariri, L. Sorbara, and K. Bizheva, "Swelling of the human cornea revealed by high speed, ultrahigh resolution optical coherence tomography," Invest. Ophthalmol. Visual Sci., published online (Apr. 30, 2010).

10. B. Young and J. W. Heath, Wheater's Functional Histology, 4th ed., Churchill Livingstone, Edinburgh, UK (2000). 